\title{
'Liking' as a First Draft of the Affective Future
}

\author{
Peter Dayan \\ MPI for Biological Cybernetics University of Tübingen \\ Tübingen, Germany
}

August 26, 2021

\begin{abstract}
The psychological and neural distinctions between the technical concepts of 'liking' and 'wanting' pose some important problems for motivated choice for goods. Why should it be that we could 'want' something that we do not 'like', or 'like' something that we would not be willing to exert any effort to acquire? Here, we suggest a framework for answering these questions through the medium of reinforcement learning. We consider 'liking' to provide immediate, but preliminary and ultimately cancellable, information about the true, long-run worth of a good. Such preliminary estimates, viewed through the lens of what is known as potential-based shaping, generally facilitate the temporally complex learning problems that animals face.
\end{abstract}

\section{Introduction}

Kent Berridge and his colleagues (Berridge, 2009a,b; Castro and Berridge, 2014; Morales and Berridge, 2020) have long argued that there is a critical difference between 'liking' and 'wanting.' 1 This distinction has been studied in greatest detail in the case of comestibles such as food and liquid; however, as we will see later. it applies more generally.

Crudely, 'liking' concerns the hedonic value of a good such as a food; whereas 'wanting' refers to the motivational force that the good can exert in terms of reorganizing the behaviour of the agent in its direction (be that by largely Pavlovian mechanisms, as in incentive sensitization, Berridge and Robinson (2016); McClure et al. (2003); or also instrumental means, Montague et al. (1996); Suri and Schultz (1999)). 'Liking', which, for comestibles in animals, is typically assessed using characteristic orofacial reactions (Berridge, 2000; Dolensek et al., 2020; Grill and Norgren, 1978), is associated with activity in what is reported as a relatively fragile network of sub-areas in the gustatory and insular cortex, the ventral striatum and the ventral pallidum, is unaffected by dopaminergic manipulations, but is modulated by opioids. By contrast, 'wanting' arises from the robust dopaminergic systems connecting midbrain, striatum and beyond.

It might seem obvious that, in untechnical terms, liking and wanting should be umbilically connected, so that we like what we want, and vice-versa. It is therefore surprising that this is apparently not always to be the case - it is often reported in the context of addiction that drugs that are keenly 'wanted' (to a significantly detrimental extent) no longer generate substantial hedonic 'liking' (Berridge and Robinson, 2016). Furthermore, neuroeconomists have delineated an even wider range of utilities (Kahneman, 1999; Kahneman et al., 1997) whose mutual divergence can lead to anomalies. Thus, along with hedonic and decision utility, which are close to 'liking' and 'wanting' respectively, are predicted utility (how much the outcome is expected to be "liked") and remembered utility

\footnotetext{
${ }^{1}$ Copying the scare quotes from papers such as Morales and Berridge (2020) to distinguish the more precise quantities that these authors have in mind from the arguably more blurry everyday meanings of these terms or subjective reports that humans can provide upon verbal request.
} 
(what one remembers about how a good was previously "liked") - and one could imagine 'wanting' versions of these latter two utilities also.

The area of food reward has cast these issues in rather stark relief (Berthoud et al., 2021; de Araujo et al., 2020). Thus recent evidence is not consistent with the idea that overconsumption and obesity (putatively consequents of over-'wanting') are increasing because of the devilishly clever 'liking'-based hedonic packaging with sweet and fat taste and texture of relatively deleterious foods (de Castro et al., 2000; Glendinning et al., 2010; Ren et al., 2010). Instead, careful experiments dissociating the oral sensory experience of foods from their gastric consequences (Bolles et al., 1981; Elizalde and Sclafani, 1990; Holman, 1969; Sclafani, 2001) suggest that it is the post-ingestive assessment by the gut of what it receives that is important for the (over-)consumption. The substrate of this involving projections via the vagus nerve ending up in the dopamine system (Fernandes et al., 2020; Han et al., 2018; Tellez et al., 2016), is quite consistent with a role in 'wanting'.

Why then indeed should we have both 'liking' and 'wanting'? In this paper, we argue that 'liking' systems play the role of what is known as potential-based shaping ( $\mathrm{Ng}$ et al., 1999) in the context of reinforcement learning (RL; Sutton and Barto, 1998). 'Liking' provides a preliminary, editable, draft version of the long-run worth of a good (Dayan, 2021). By providing an early guess at a late true value, this can help with the notorious temporal credit assignment problem in RL (Sutton and Barto, 1998), which stems from the fact that, in most interesting domains, agents and animals alike have to wait for a long period of time and/or make whole sequences of choices before finding out, much later, whether these were appropriate.

These preliminary, draft, hedonic values thus steer animals towards what will normally be appropriate choices - making learning operate more effectively. RL borrowed the term 'shaping' from psychology (Krueger and Dayan, 2009; Skinner, 1938, 1975) to encompass a number of methods for improving the speed and reliability of learning - just like the effect we are arguing for here. One class of methods systematically adds quantities to 'true' underlying rewards; however, like many methods that manipulate utilities, unintended consequences are rife. Potential-based shaping was suggested by $\mathrm{Ng}$ et al. (1999) as a variant that is guaranteed not to have such consequences, and indeed is equivalent to a typically optimistic initialization of the estimation of values (Wiewiora, 2003).

In the case of victuals: for survival, animals actually care about the nutritive value of foods (which is why they underpin 'wanting') - this is the long run worth. However, it takes time for the digestive system to process these foods to determine their underlying value, making it difficult to criticize or reward the actions that led to them in the first place. This is exactly the temporal credit assignment problem. Instead, exteroceptive sensory input from the mouth and nose (and even the visual system) underpins a guess at this true value - providing immediate hedonic feedback for the choice of action. Usually, this guess is good, and so the two systems harmonize seamlessly. Given disharmony, it is the nutritive value that should determine ultimate choice, as described above. Thus, even if the orofacial 'liking' responses might themselves not be manipulated by 'wanting' system substrates such as dopamine, it is by activating dopamine systems in particular patterns that hedonic value can act appropriately.

We first describe conventional model-free methods for prediction in RL, and the role of potential-based shaping in this. We then use the case of flavour-nutrient conditioning to suggest how the systems concerned might interact. Finally, in the discussion, we touch on some more speculative suggestions about the underlying source of utility in the context of 
homestatic RL (Keramati and Gutkin, 2014) and discuss a version of the same argument, but now for aesthetic value (Brielmann and Dayan, 2021).

\section{Model-free RL}

In the main part of this paper, we concentrate on Pavlovian conditioning (Mackintosh, 1983) - the case in which predictions about future, potentially valuable outcomes lead to automatic actions such as approach, engagement and even licking (whether or not those actions are actually useful for acquiring those outcomes; Dayan et al., 2006). Thus, we focus on problems of evaluation; and save consideration of the choice between actions for later.

We consider a Markov prediction problem in a terminating, episodic, case with no temporal discounting. Here, there are connected states, $s \in \mathcal{S}$, a special terminating state $s^{*}$, a transition matrix $\mathcal{T}_{s s^{\prime}}=P\left(s_{t+1}=s^{\prime} \mid s_{t}=s\right),\left\{s, s^{\prime}\right\} \in \mathcal{S}$, with $\tilde{\mathcal{T}}_{s}=1-\sum_{s^{\prime} \in \mathcal{S}} \mathcal{T}_{s s^{\prime}}=$ $P\left(s_{t+1}=s^{*} \mid s_{t}=s\right)$ and rewards $r_{s} \in \Re$ associated with state $s$ (which we will assume to be deterministic for convenience; also writing vector $\mathbf{r}$ for the rewards for all states); and $r_{s^{*}}=0$.

Then, if we write $V_{s}=E_{s_{1}=s}\left[\sum_{t=1}^{\infty} r_{s_{t}}\right]$ for the long run value of state $s \in \mathcal{S}$ (the value of $s^{*}$ is 0 ), and vector $\mathbf{V}$ for all the values, we have

$$
\begin{aligned}
& V_{s}=r_{s}+\sum_{s^{\prime}} \mathcal{T}_{s s^{\prime}} V_{s^{\prime}} \quad \text { or } \\
& \mathbf{V}=\mathbf{r}+\mathcal{T} \mathbf{V}=[\mathcal{I}-\mathcal{T}]^{-1} \mathbf{r}
\end{aligned}
$$

by writing the recursion directly.

The simplest form of temporal difference (TD) learning (Sutton, 1988; Sutton and Barto, 1998) attempts to learn the values $V_{s}$ from stochastic trajectories $s_{1}, s_{2}, s_{3}, \ldots, s^{*}$ generated by sampling from $\mathcal{T}$. TD accomplishes this by constructing a prediction error from the sampled difference between right and left side of equation 1

$$
\delta_{t}=r_{s_{t}}+V_{s_{t+1}}-V_{s_{t}}
$$

and applying

$$
V_{s_{t}}=V_{s_{t}}+\alpha \delta_{t}
$$

where $\alpha$ is the learning rate. There is substantial evidence that the phasic activity of at least some dopamine neurons in the ventral tegmental area of the midbrain, and the release of dopamine in target regions such as the nucleus accumbens, reports the temporal difference prediction error $\delta_{t}$ of equation 3 (Cohen et al., 2012; Hart et al., 2014; Kim et al., 2020; Kishida et al., 2016; Montague et al., 1996; Schultz et al., 1997).

In cases of instrumental conditioning, when actions must also be chosen, the prediction error $\delta_{t}$ can also be used to critize a choice (in an architecture called the actor-critic; Barto et al., 1983). The idea is that actions that lead either to unexpected good rewards (judged by $r_{s_{t}}$ ) or unexpectedly good states (judged by large expected long-run future rewards, $V_{s_{t+1}}$ ) should be more likely to be chosen in the future. This can be measured by $\delta_{t}$.

Although temporal difference learning is powerful, offering various guarantees of convergence when the learning rate $\alpha$ satifies suitable conditions, it has the problem of being 
A

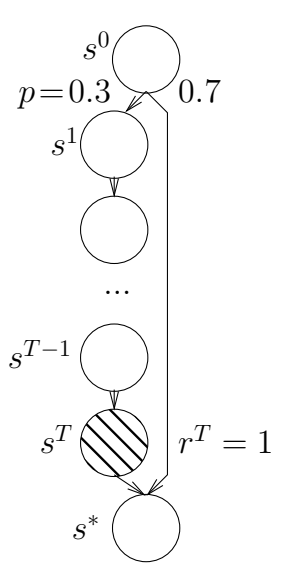

B
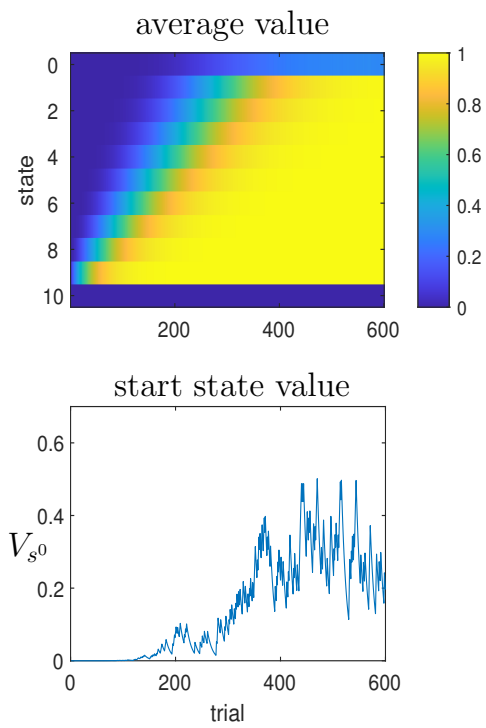

C
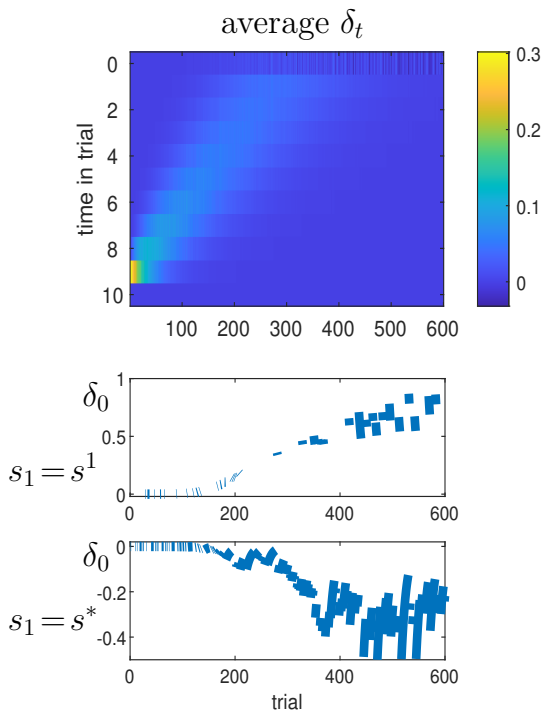

Figure 1: TD-based Markov prediction. A) Simple Markov prediction problem with a tasty morsel provided at $t=1\left(s=s^{1}\right)$ with probability $p=0.3$; which leads to a digestive reward of $r^{=} 1$ at time $T$. B) Evolution of the Value for the application of TD learning to the case that $T=10$. Upper plot: average over many simulations; lower plot: single simulation showing $V_{s^{0}}$. C) Evolution of the TD prediction error $\delta_{t}$ over the same trials. Upper plot: average over many simulations; lower plots: single simulation showing $\delta_{0}$ for a transition to $s=s^{1}$ (above); or to $s=s^{*}$ (below). Here $\alpha=0.1$.

sometimes slow. To illustrate this, we consider a case related to the one that we will consider later in flavour-nutrient conditioning. Figure $1 \mathrm{~A}$ shows a case in which from a start state $s=s^{0}$ (written as just 0 , since we can identify this with time within the trial), there is a high probability $(p=0.7)$ transition directly to the terminal state $s^{*}$, and a low probability transition to state $s=s^{1}$, associated with an observation (later modeling the oral sensation of a morsel of food) and which initiates a sequence of $T$ states leading to a rewarding outcome $r^{T}=1$ (later modeling the gut's evaluation of this morsel) and then the terminal state $s^{*}$.

The left plots in figure 1B depict the course of learning of the value of the value structure associated with selected states, applying equations 3 and 4 . The upper plot depicts the average value (across many simulations) for all non-terminal states as a function of learning trial. As expected for this sort of complete serial compound stimulus representation (Gershman et al., 2014; Ludvig et al., 2012) in which every timestep following the morsel of food is separately individuated, the value of the reward available at $s^{T}$ apparently propagates backwards to $s^{1}$. The further propagation to $s^{0}$ is then affected by the stochasticity at that single state. The lower plot shows the evolution of $V_{s^{0}}$ for one single run; the slow rise and stochastic fluctuations are evident.

The right plots in figure $1 \mathrm{~B}$ show the prediction errors that occasion the learning of the values shown in the left plots. For convenience, in the single example beneath, we have separated the case that the transition from $s^{0}$ is to $s^{1}$, and ultimately to the actual reward at $s^{T}$ (upper) from the case that the transition is to $s^{*}$, and thus no reward (lower). Given that the average value of $V_{s^{0}}=p=0.3$, the former transition is associated with a positive prediction error; the latter with a negative one. Note that at the end of learning, the only prediction error arises at time $t=0$, because of the stochasticity associated with the 
transition to $s^{1}$ versus $s^{*}$. At all other states, predictions are deterministically correct.

The most salient characteristic of the learning in this case is its sloth - apparent in the averages and the single instance. There are two reasons for this: first $p$ is low, which means that the agent usually fails to sample $s^{1}$ and the cascade leading to the reward. The second is that the learning rate $\alpha=0.1$ is rather modest. Increasing $\alpha$ leads to faster learning, but also greater fluctuations in the values and prediction errors.

\section{Potential-based shaping}

Shaping was originally suggested in the context of policy learning as a way of leading subjects through a sequence of steps in order to facilitate learning of good performance in a particular task (Skinner, 1975). The idea is to provide a set of intermediate (typically state- and/or action-dependent) rewards that are different from those specified by the task itself in order to provide an easier path for animals to learn appropriate final behaviour. The benefit of this has also been recognized in RL (e.g., Ng et al., 1999; Randløv and Alstrøm, 1998), also leading to ideas about intrinsic rewards (Sorg et al., 2010, by contrast with the extrinsic rewards that are determined by the task).

Citing entertaining examples such as the micro-circling bicycle of Randløv and Alstrøm (1998), Ng et al. (1999) observed that manipulating the reward structure ( $r_{s}$ in our terms) can have unintended consequences - skewing predictions (and, in instrumental cases, choices) away from their optimal values for the original task. They therefore suggested a scheme called potential-based shaping which could steer learning, but with a guarantee of no asymptotic effect. This involves adding a function of state $\phi_{s}$ to temporal difference error terms such as that in equation 3 , making it

$$
\delta_{t}=r_{s_{t}}+\left[\phi_{s_{t+1}}-\phi_{s_{t}}\right]+V_{s_{t+1}}-V_{s_{t}} .
$$

The name potential-based shaping comes from the fact that summing the net effect of $\phi$ in cycles of states is 0 , because it appears in difference form - thus it satisfies the same sort of no-curl condition as a conventional potential function. This means that it does not distort the values ascribed to states at the asymptote of learning when the predictions have converged. However, the idea is that the shaping function provides a hint about the values of states - being large for states that are associated with large long run reward. Thus, a transition from a state $s_{t}=s$ to $s_{t+1}=s^{\prime}$ when $\phi_{s}$ is low and $\phi_{s^{\prime}}$ is high will provide immediate error information to the value $V_{s}$ for state $s$ even if $V_{s^{\prime}}=0$ has not yet been learned. In an instrumental conditioning case, the resulting high value of $\delta_{t}$ will also be useful information that the action just taken that led to this reward and transition is also unexpectedly good and so is worth favoring.

For the Markov prediction problem of figure 1, the appropriate shaping function associated with the morsel of food is rather straightforward - it should be $\phi_{s}=1$ for $s=s^{1} \ldots s^{T-1}$ and $\phi_{s^{T}}=0$. The reason is that ingestion of the morsel with its sweet taste (at $s^{1}$ ) predicts the benefit of digestion (at $s^{T}$ ) for all those future times. Formally, the hedonic value is generated by $\phi_{s_{t+1}}-\phi_{s_{t}}$.

Figure 2 shows the course of learning in the Markov prediction problem of figure 1 given thise perfect shaping function (shown in figure 2A). It is apparent that acquisition of the correct value for $V_{s^{0}}$ is greatly accelerated, as is the advent of the correct set of prediction errors (which are immediately zero for $s \neq s^{0}$ ). This shows the benefit of shaping. The 
A

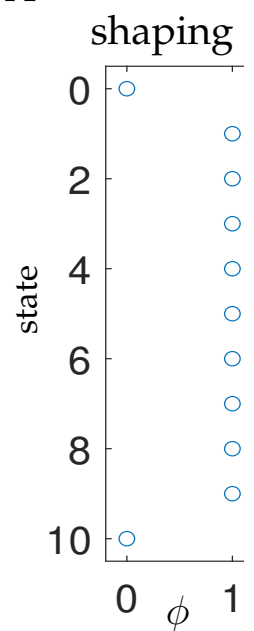

$\mathrm{B}$
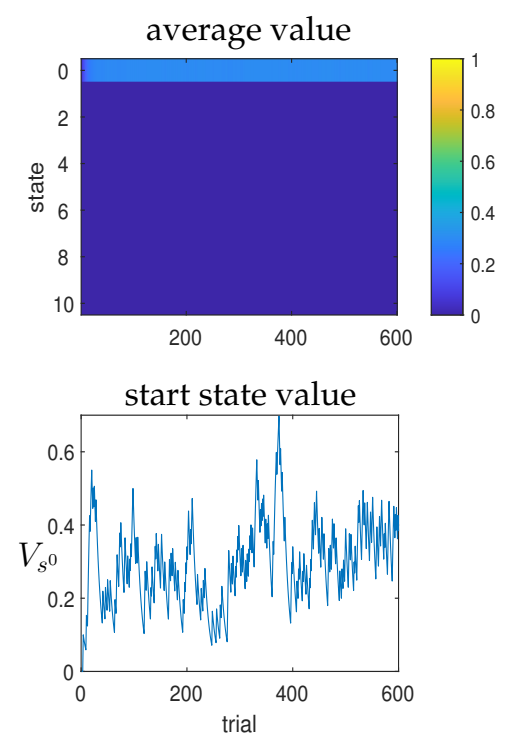

C
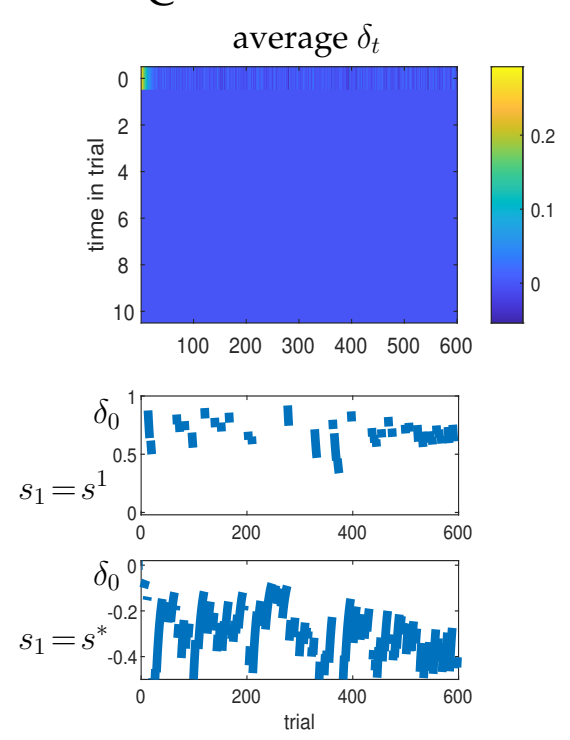

Figure 2: TD-based Markov prediction with perfect shaping. A) The ideal shaping function $\phi$ is 1 after acquisition of the food $\left(\right.$ at $\left.s^{1}\right)$. B) Evolution of the Value for the application of TD learning to the case that $T=10$. Upper plot: average over many simulations; lower plot: single simulation showing $V_{s^{0}}$. C) Evolution of the TD prediction error $\delta_{t}$ over the same trials. Upper plot: average over many simulations; lower plots: single simulation showing $\delta_{0}$ for a transition to $s=s^{1}$ (above); or to $s=s^{*}$ (below). Here $\alpha=0.1$.

agent can learn quickly that the state giving access to the morsel of food is itself appetitive. Furthermore, in a more complex problem in which there is a choice between actions, one of which provided access to $s^{0}$, this action could also be learned as being worth 0.3 units of reward.

Note also an important difference between figures 1 and 2 - namely that, at the end of learning, $V_{s}=0$ for $s=s^{\tau}, \tau \geq 1$ in the latter, but not the former. The reason for this is that the prediction error is 0 for $t \neq 0$ because of the perfection of the shaping function - implying that there is nothing to learn for the states that lie between ingestion and digestion. In fact, the total prediction of the long run reward from a state is $V_{s}+\phi_{s}$. It has thus also been observed that a perfect substitute for this sort of potential-based shaping is to initialize $V_{s}=\phi_{s}$, and then use standard temporal difference learning, as in equations 3 and 4 (Wiewiora, 2003). However, although this is technically correct, it is not suitable for our purposes of flavor-nutrient conditioning since it does not respect a separation between taste processing and conditioning mechanisms.

If the shaping function $\phi_{s}$ is not perfect, then the course of learning will be at least partially disrupted. Figure 3 shows a case in which the shaping function decays linearly from $\phi_{s^{1}}=1$ to $\phi_{s^{T-1}}=0$, as if the prediction from the taste system associated with the future digestive benefit cannot last as long as the time that the gut takes to process the food morsel. In this case, the prediction $V_{s^{0}}$ learns very quickly at first, but then temporarily decreases modestly (from around trials 50-200 in the example) before recovering. The suppression arises since $\delta_{t}<0$ for $t=1 \ldots T-1$ on early learning trials (since $\phi_{s^{t}}$ is decaying linearly over these times), and this negative prediction error propagates backwards to influence $V_{0}$. Later, the positive prediction error that starts associated with the digestive report of the nutritive value (i.e., $r^{T}=1$ ) itself propagates back to overwhelm the suppression. Furthermore, the asymptotic value $V_{s}$ comes over the course of learning 
A

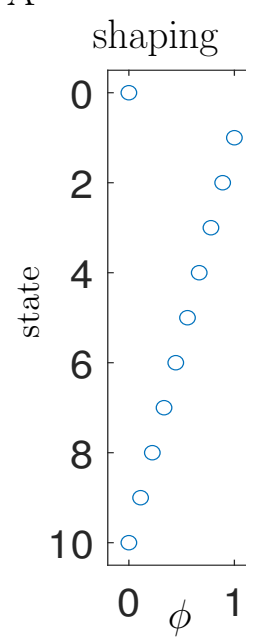

$\mathrm{B}$
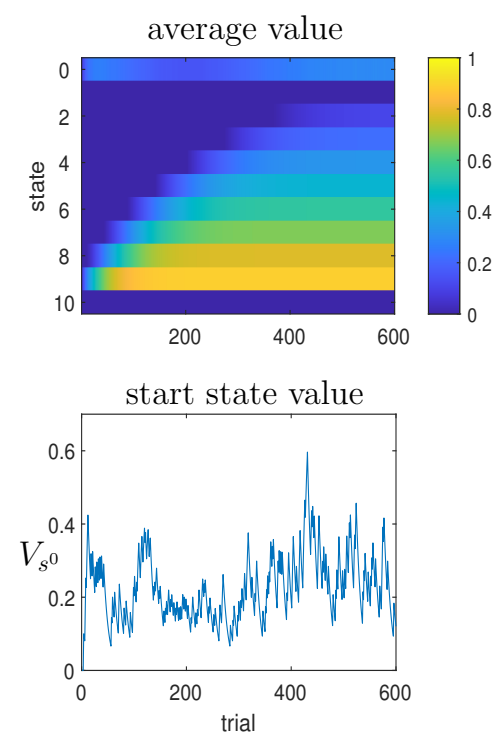

C
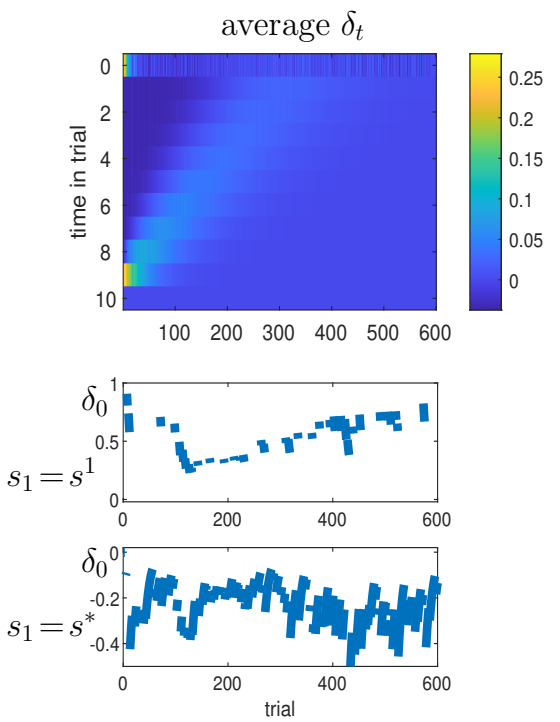

Figure 3: TD-based Markov prediction with a partial shaping function. A) A suboptimal shaping function $\phi$ that decreases from 1 to 0 linearly after acquisition of the food (at $\left.s^{1}\right)$. B) Evolution of the Value for the application of TD learning to the case that $T=10$. Upper plot: average over many simulations; lower plot: single simulation showing $V_{s^{0}}$. C) Evolution of the TD prediction error $\delta_{t}$ over the same trials. Upper plot: average over many simulations; lower plots: single simulation showing $\delta_{0}$ for a transition to $s=s^{1}$ (above); or to $s=s^{*}$ (below). Here $\alpha=0.1$.

exactly to compensate for the inadequacy of the shaping function such that $V_{s}+\phi_{s}$ is the long-run reward from state $s$.

\section{Flavour-nutrient conditioning}

Flavour-nutrient conditioning has a venerable history (Berthoud et al., 2021; de Araujo et al., 2020; Elizalde and Sclafani, 1990; Mehiel and Bolles, 1984, 1988; Myers, 2018; Sclafani, 2004). The idea is to separate the impact of any immediate sensory input associated with a liquid or food: taste, smell, sight, oral texture, whisker responses, and the like from what is sometimes known as its post-oral effects - the results of processing in the stomach, gut and beyond. The key questions are which of these drives 'liking' and 'wanting' for the consumable.

One of the most popular methods is to use a form of so-called electronic oesophagus (Elizalde and Sclafani, 1990). With this, an animal can be allowed to sample substances orally by licking them; but the licks are paired with the delivery of a potentially different substance directly into the stomach of an animal through a catheter. Thus, it is possible to dissociate fully the various sensory qualities of an ingestible substance from its digestible nutrient content and to assess issues such as an animal's ability to learn about the relationship between an originally neutral flavour and appetitive or aversive digestive consequences.

The result of an extensive body of work in this direction is quite consistent with the separation between 'liking' and 'wanting' (Morales and Berridge, 2020). The immediate hedo- 
nic quality of consumables, associated with 'liking', is assessed rapidly by exteroceptive sensory systems based on connections to primary and sensory taste cortex, amygdala, insular cortex, and beyond. The influence of consumables on long-run consumption (and motivational attraction), associated with 'wanting', is assessed more slowly by interoceptive mechanisms, with ultimate connections via the vagus nerve (and possibly dorsal root ganglia) to the dopamine system (Fernandes et al., 2020; Han et al., 2018; Tellez et al., 2016). Thus, although pairing food with subsequent sickness (including gastric malaise) has a powerful impact on dis-'liking' (Garcia et al., 1985), and there is some evidence (e.g., Myers and Sclafani, 2001) for an original expectation that advantageous interoceptive discoveries about the nutritive quality of foods or liquids with novel tastes would exert its effect by increasing the hedonic pleasure of those tastes, the extent of this latter change pales in comparison with one associated with a separate and powerful form of 'wanting' (Myers, 2018). This asymmetry is perhaps in keeping with the reported fragility of the 'liking' system (Berridge and Robinson, 2016).

In order to illustrate the effect of paradigms in which exteroceptive and interoceptive qualities are orthogonalized, we simulated a version of the shaping paradigm described in the previous section, but with foods of three separate flavours associated with three different nutritive values. One (shown by the red line in figure 4) is not at all sweet, and so lacks any shaping reward; but is highly nutritious. A second (green) is very sweet, attracting substantial shaping; but lacks any nutritive value; the final flavour (blue) is of intermediate sweetness and nutrition - but in a way that matches (at least given the prevailing motivational state; Cabanac, 1971). Here, for convenience, we consider a deterministic case in which each flavour is treated separately, and with a faster learning rate than in the previous section $(\alpha=0.4)$.

Figure 4 shows the course of learning of the value that is assigned to each of the three flavours over the course of exposures. To our knowledge, this particular experiment has not been performed; so these quantities could be seen as predictions of relative preference in an appropriate test of choice. The purely nutritive, non-sweet, flavour (red) only gains value slowly, but ultimately reaches a high asymptote. Learning is delayed without the benefit of shaping. The purely sweet, non-nutritive, flavour (green) becomes attractive very rapidly, because it outwits the shaping mechanism. However, ultimately, the nutritive value dominates, and so its ultimate value reduces to 0 . Finally, the conventional, modestly-appetitive flavour (blue), shows the fast time-course of learning evident also in the previous section, since the shaping function is correct - with 'liking' and 'wanting' being aligned.

\section{Discussion}

In this paper, we provided a reinforcement learning (RL) view of 'liking' and 'wanting' which uses the construct of potential-based shaping (Ng et al., 1999) as a basis for a hedonic signal inspired by a sensory object. This steers an RL rule such as temporal difference learning when veridical information about the long-run worth of that object may arise only slowly.

We illustrated this argument using the modern conceptions of flavour-nutrient conditioning, because some of the most extensive data and discussions on the distinction between 'liking' and 'wanting' have arisen in this domain. Here, 'liking' provides a preliminary assessment of the long-run worth of a morsel of food or a drop of liquid. The latter is 


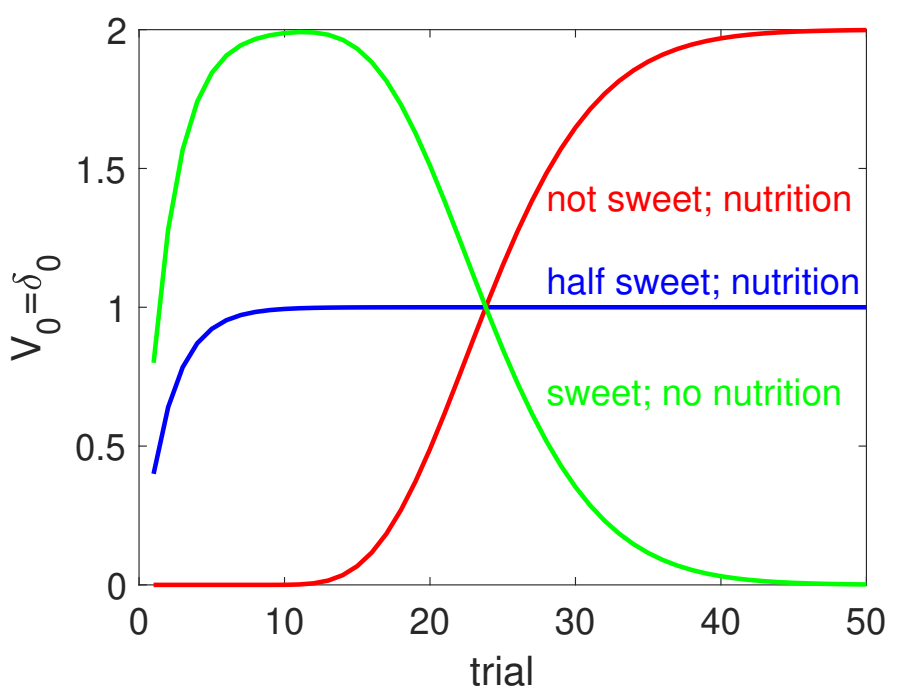

Figure 4: Simulation of flavour-nutrient conditioning. The lines show the evolution of the learned value of three different flavours with orthogonalized intrinsic sweetness and nutritive values. The red flavour is not sweet, but is highly nutritious - and so lacks shaping (as in figure 1). The green flavour is very sweet (with a shaping function reflecting this), but is not nutritious. The blue flavour is somewhat sweet and somewhat nutritious, and is also associated with a perfect shaping function (as in figure 2). Here, transitions are deterministic and $\alpha=0.4$.

ultimately reported by post-oral evaluation mechanisms feeding into the dopamine system, and is the substrate for establishing the motivational impact or 'wanting' for those foodstuffs.

The requirements on 'liking' to be perfectly aligned with 'wanting' are relatively stringent. One necessity is for an assessment of the long-run value to be made based on the very distal information provided through oral evaluation. This is particularly hard in the era of processed foods (and artificial sweeteners, e.g., Veldhuizen et al., 2017), as methods for making prestidigitators out of taste and olfactory receptors abound. There is also at least one very prominent converse anomaly: fructose, which is actually sweeter to the taste than glucose at equivalent concentrations, and is a key raw material for lipogenesis in the liver (thus having advantageous digestive import), apparently fails to generate substantial post-oral 'wanting' signals (Myers et al., 2020). The full range of learning anomalies to which this leads has yet to be mined. Furthermore, there are various experimental procedures which can make persistently dis-'liked' goods strongly 'wanted' (Myers and Sclafani, 2003; Warlow et al., 2020).

One important and controversial set of subtleties on which the literature does not seem completely settled is the precise roles of dopamine in these processes (Berridge, 2007; Cameron et al., 2017; McCutcheon, 2015; Salamone and Correa, 2013). This is important because of dopamine's multi-faceted role in appetitive learning - including representing the sort of phasic temporal difference prediction error for reward that we wrote in equation 3 (Montague et al., 1996; Schultz et al., 1997), and a more tonic representation of the reward availability in an environment (Hamid et al., 2016; Niv et al., 2007). One source of complexity is the potentially differential role of different parts and projections of the dopamine system - notably the ventral tegmental area (VTA) and the substantia nigra pars compacta $(\mathrm{SNc})$, connected respectively with the ventral and dorsal striatum, and often implicated respectively (though perhaps incompletely; Hamid et al., 2021) in value 
learning (and Pavlovian responses) and action learning (Montague et al., 1996; Suri and Schultz, 1999).

There is excellent evidence that dopamine is not involved in the orofacial reactions that are taken as the ground truth for 'liking', but that it is deeply implicated in 'wanting' (Berridge, 2007; Berridge and Robinson, 1998). However, non-nutritive but orally-attractive sweeteners such as sucralose do lead to the activity of dopamine neurons (e.g., Fernandes et al., 2020); and Tellez et al. (2016) reported that the release of dopamine into the ventral (but not dorsal) striatum was identical for sucralose and sucrose. This would be consistent with the potential-based shaping that we have described. However, while Tellez et al. (2016) and Han et al. (2018) reported a separation between hedonic and nutritive aspects of sucrose, with a critical role only for dopamine in the dorsal striatum (and a pathway to this associated with neurons in the right nodose ganglion of the right side of the vagus nerve), Fernandes et al. (2020) reported that the post-ingestive import of sucrose (relative to sucralose) is mediated, at least in substantial part, by connections running via the left side of the vagus nerve (the left nodose ganglion) to VTA dopamine neurons (and thus presumably the ventral striatum), and that it is activation of this pathway that can sustain vigorous operant behaviour.

These subtleties mainly concern the alignment of 'liking' and 'wanting' in terms of value. The other aspect of alignment, highlighted by figure 3 concerns timing. An optimal shaping function would remain high for the whole length of time until the report of the 'true' worth of a sensory object is available. This is hard by itself; and maintaining information about which object inspired which later signal of true worth would seem impossible. Indeed, paradigms in which nutritive and non-nutritive pellets of food are provided less than two minutes apart show that the ascription of pellet to consequence can be rendered highly imperfect McCutcheon et al. (2012). It would be interesting to examine whether hedonic systems can sustain relatively more tonic activity (noting that, under the shaping hypothesis, this might not be apparent in the activity of dopamine neurons (since, as evident in figure 1 , the prediction error becomes 0 ).

One wider context for this work is a progressive blurring within RL of the understanding of utility and reward as being defined by the environment versus the animal or agent itself (Eschmann, 2021; Keramati and Gutkin, 2014; Oudeyer et al., 2007; Singh et al., 2009). One prominent example of this is the field of homeostatic RL (Keramati and Gutkin, 2014), which starts from the oddly frequently overlooked point that the environment does not provide any external evaluation even of primary reinforcers (such as food or liquid). Instead, through reproductive sorting, evolution has programmed a set of internal mechanisms for evaluating primary reinforcers that have been found, historically, to benefit fitness. Keramati and Gutkin (2014) formulated this problem via the notion of an optimal homeostatic set point for various physiologically-important variables in a complex internal state space, plus the suggestion that internal utility is generated by movement relative to this set point. In a form of generalized drive reduction theory (Hull, 1943), movement towards the set point (reducing the drive for the aspects of the state that are dysregulated) would be appetitive, associated with positive reward $r$; movement away would be aversive, associated with negative reward $r$.

The potential-based shaping version of this (Dayan, 2021) suggests that, indeed there is no external reward at all $r_{t}=0, \forall t$. However, instead, evolution has endowed us with a large-scale shaping function that nominally estimates a scalar quantity of external semantics and significance, such as expected lifetime - from information about internal state (of nutrition, hydration and the like) (Petzschner et al., 2021). Then changes in 
internal state that increase or decrease lifetime generate positive or negative contributions respectively to the prediction error of equation 5, and substitute for external reward. Alternatively, we could have been endowed directly with what amounts to the derivative of this function $\phi_{s_{t+1}}-\phi_{s_{t}}$, which is the only way that the shaping function appears in practice.

In terms of the argument in our paper, there could be both cortical ('high road'; putatively involving areas such as the insular cortex) and sub-cortical ('low-road') such shaping functions or derivatives which respond to physiological signals (de Araujo et al., 2020). Thus, we would generalize from a hedonic-based shaping function (from exteroceptive sensation) coupled to a ground-truth reward function associated with nutritive properties to the sum of two different shaping functions - a hedonic, 'liking'-associated, exteroceptive one, and a ground-truth, 'wanting'-associated, interoceptive one. One can imagine further generalizing such a scheme.

A second example comes from a recent theory for the basis of aesthetic value for sensory objects such as pictures (Brielmann and Dayan, 2021). The idea is that an animal should ideally have a sensory system that is efficient at performing information processing on the distribution of sensory inputs that it is likely to experience in the future. This efficiency is commonly defined in the Helmholtzian terms of the fit of an internal generative model to this distribution (Hinton and Sejnowski, 1999), implying that a suitable shaping function based on the state of the sensory processing system is a measure of this fit (the Kullback-Liebler divergence, or average log probability, in Brielmann and Dayan, 2021).

In one version of their account, the effect of observing a sensory input is two-fold: one form of learning changes the sensory system to increase the probability that this input would be generated; and a second is associated with an increase in the probability of this particular input in the likely distribution of future stimuli. Consider following the proposal in the current paper of regarding the change in shaping function as generating hedonic 'liking'. The first component then realizes one popular form of aesthetic value associated with learning progress or prediction error (Schmidhuber, 2010) - rewarding the change of the sensory system associated with the input. If changes to the sensory system associated with any individual input are modest, the second component amounts to rewarding stimuli that are likely under the generative model - a factor closely related to the fluency with which the input is processed, which is a second, and traditionally competing, popular theory for aesthetic value (Reber et al., 2004; Ryali et al., 2020; Van de Cruys and Wagemans, 2011). Thus a potential-based shaping theory of 'liking' unifies these two concepts of aesthetic value. How the equivalent of 'wanting' is calculated or represented is less clear.

Based originally on some gustatory Italian misadventures, Dickinson and Balleine (2010) have suggested an incentive learning view of 'liking' as an hedonic interface between two 'psychologies': a way of instructing a goal-directed instrumental control system about the 'true', bodily value of an affectively-charged outcome. In RL terms, this would be a way by which a model-based system (Daw et al., 2005; Dolan and Dayan, 2013) could help decide which goals are potentially worth pursuing. However, although there clearly are cases such as food aversion learning in which dis-'liking' is the consequence of a somatic evaluation of an outcome (Garcia et al., 1985), we have seen that this is not ubiquitous, with 'wanting' better reflecting the ultimate worth. Of course, the more accurately 'liking' reflects a true current value, the better the shaping signal it can provide; it seems, however, that this is incomplete. The form of involvement of the dopamine system in 'wanting' is rather suggestive of model-free control. However, the paradigms we have 
discussed do not provide clear evidence about the extent to which when 'wanting' separates from 'liking', the 'wanting' value can influence model-based control, as it normatively should.

In sum, we have provided an account of 'liking' in terms of a reinforcement learning theory of potential-based shaping. We used the example of flavour-nutrient conditioning to show how 'liking' could be aligned with 'wanting', and to show some of its desirable properties in terms of speeding learning when this happens. We also noted links with homeostatic RL, where multiple layers of extero- and intero-ceptive shaping might be combined, and to the hedonics associated with sensory aesthetics. 'Liking' amounts to a loan that, provided it is paid back in a timely manner by processes associated with 'wanting', will organize the smooth coordination of learning and behaviour.

\section{Acknowledgments}

I am very grateful to Ivan de Araujo, Kent Berridge, Aenne Brielmann, Mehdi Keramati, Kevin Lloyd, Read Montague and Dana Small for historical and contemporary discussions on many aspects of this work. Funding was from the Max Planck Society and the Alexander von Humboldt Foundation.

\section{References}

Barto, A. G., Sutton, R. S., and Anderson, C. W. (1983). Neuronlike adaptive elements that can solve difficult learning control problems. IEEE transactions on systems, man, and cybernetics, 13:834-846.

Berridge, K. C. (2000). Measuring hedonic impact in animals and infants: microstructure of affective taste reactivity patterns. Neuroscience E Biobehavioral Reviews, 24(2):173-198.

Berridge, K. C. (2007). The debate over dopamine's role in reward: the case for incentive salience. Psychopharmacology, 191(3):391-431.

Berridge, K. C. (2009a). Wanting and liking: Observations from the neuroscience and psychology laboratory. Inquiry, 52(4):378-398.

Berridge, K. C. (2009b). 'Liking'and 'wanting' food rewards: Brain substrates and roles in eating disorders. Physiology $\mathcal{E}$ behavior, 97(5):537-550.

Berridge, K. C. and Robinson, T. E. (1998). What is the role of dopamine in reward: hedonic impact, reward learning, or incentive salience? Brain research reviews, 28(3):309-369.

Berridge, K. C. and Robinson, T. E. (2016). Liking, wanting, and the incentivesensitization theory of addiction. The American psychologist, 71:670-679.

Berthoud, H.-R., Morrison, C. D., Ackroff, K., and Sclafani, A. (2021). Learning of food preferences: mechanisms and implications for obesity \& metabolic diseases. International Journal of Obesity, pages 1-13.

Bolles, R. C., Hayward, L., and Crandall, C. (1981). Conditioned taste preferences based on caloric density. Journal of Experimental Psychology: Animal Behavior Processes, 7(1):59. 
Brielmann, A. A. and Dayan, P. (2021). Introducing a computational model of aesthetic value. In submission.

Cabanac, M. (1971). Physiological role of pleasure. Science, 173(4002):1103-1107.

Cameron, J. D., Chaput, J.-P., Sjödin, A. M., and Goldfield, G. S. (2017). Brain on fire: Incentive salience, hedonic hot spots, dopamine, obesity, and other hunger games. $A n$ nual Review of Nutrition, 37:183-205.

Castro, D. and Berridge, K. C. (2014). Advances in the neurobiological bases for food 'liking'versus 'wanting'. Physiology \& behavior, 136:22-30.

Cohen, J. Y., Haesler, S., Vong, L., Lowell, B. B., and Uchida, N. (2012). Neuron-typespecific signals for reward and punishment in the ventral tegmental area. nature, 482(7383):85-88.

Daw, N. D., Niv, Y., and Dayan, P. (2005). Uncertainty-based competition between prefrontal and dorsolateral striatal systems for behavioral control. Nature neuroscience, 8(12):1704-1711.

Dayan, P. (2021). When will's wont wants wanting. Behavioral and Brain Sciences, 44.

Dayan, P., Niv, Y., Seymour, B., and Daw, N. D. (2006). The misbehavior of value and the discipline of the will. Neural networks, 19(8):1153-1160.

de Araujo, I. E., Schatzker, M., and Small, D. M. (2020). Rethinking food reward. Annual review of psychology, 71:139-164.

de Castro, J. M., Bellisle, F., Dalix, A.-M., and Pearcey, S. M. (2000). Palatability and intake relationships in free-living humans: characterization and independence of influence in north americans. Physiology \& behavior, 70(3-4):343-350.

Dickinson, A. and Balleine, B. (2010). Hedonics: the cognitive-motivational interface. In Kringelbach, M. L. and Berridge, K. C., editors, Pleasures of the Brain, pages 74-84. Oxford University Press New York, NY.

Dolan, R. J. and Dayan, P. (2013). Goals and habits in the brain. Neuron, 80(2):312-325.

Dolensek, N., Gehrlach, D. A., Klein, A. S., and Gogolla, N. (2020). Facial expressions of emotion states and their neuronal correlates in mice. Science, 368(6486):89-94.

Elizalde, G. and Sclafani, A. (1990). Flavor preferences conditioned by intragastric polycose infusions: a detailed analysis using an electronic esophagus preparation. Physiology E Behavior, 47(1):63-77.

Eschmann, J. (2021). Reward function design in reinforcement learning. Reinforcement Learning Algorithms: Analysis and Applications, pages 25-33.

Fernandes, A. B., da Silva, J. A., Almeida, J., Cui, G., Gerfen, C. R., Costa, R. M., and Oliveira-Maia, A. J. (2020). Postingestive modulation of food seeking depends on vagus-mediated dopamine neuron activity. Neuron, 106(5):778-788.

Garcia, J., Lasiter, P. S., BERMUDEZ-RATTONI, F., and Deems, D. A. (1985). A general theory of aversion learning a. Annals of the New York Academy of Sciences, 443(1):8-21.

Gershman, S. J., Moustafa, A. A., and Ludvig, E. A. (2014). Time representation in reinforcement learning models of the basal ganglia. Frontiers in computational neuroscience, $7: 194$. 
Glendinning, J. I., Beltran, F., Benton, L., Cheng, S., Gieseke, J., Gillman, J., and Spain, H. N. (2010). Taste does not determine daily intake of dilute sugar solutions in mice. American Journal of Physiology-Regulatory, Integrative and Comparative Physiology, 299(5):R1333-R1341.

Grill, H. J. and Norgren, R. (1978). The taste reactivity test. i. mimetic responses to gustatory stimuli in neurologically normal rats. Brain research, 143(2):263-279.

Hamid, A. A., Frank, M. J., and Moore, C. I. (2021). Wave-like dopamine dynamics as a mechanism for spatiotemporal credit assignment. Cell, 184(10):2733-2749.

Hamid, A. A., Pettibone, J. R., Mabrouk, O. S., Hetrick, V. L., Schmidt, R., Vander Weele, C. M., Kennedy, R. T., Aragona, B. J., and Berke, J. D. (2016). Mesolimbic dopamine signals the value of work. Nature neuroscience, 19(1):117-126.

Han, W., Tellez, L. A., Perkins, M. H., Perez, I. O., Qu, T., Ferreira, J., Ferreira, T. L., Quinn, D., Liu, Z.-W., Gao, X.-B., Kaelberer, M. M., Bohórquez, D. V., Shammah-Lagnado, J., Lartiguel, G., and de Araujo, I. E. (2018). A neural circuit for gut-induced reward. Cell, 175(3):665-678.

Hart, A. S., Rutledge, R. B., Glimcher, P. W., and Phillips, P. E. (2014). Phasic dopamine release in the rat nucleus accumbens symmetrically encodes a reward prediction error term. Journal of Neuroscience, 34(3):698-704.

Hinton, G. E. and Sejnowski, T. J., editors (1999). Unsupervised learning: foundations of neural computation, Cambridge, MA. MIT Press.

Holman, G. L. (1969). Intragastric reinforcement effect. Journal of comparative and physiological psychology, 69(3):432.

Hull, C. L. (1943). Principles of behavior: An introduction to behavior theory. AppletonCentury.

Kahneman, D. (1999). Objective happiness. In Kahneman, D., Diener, E., and Schwarz, N., editors, Well-being: The foundations of hedonic psychology, pages 1-23. Russell Sage Foundation.

Kahneman, D., Wakker, P. P., and Sarin, R. (1997). Back to bentham? explorations of experienced utility. The quarterly journal of economics, 112(2):375-406.

Keramati, M. and Gutkin, B. (2014). Homeostatic reinforcement learning for integrating reward collection and physiological stability. Elife, 3:e04811.

Kim, H. R., Malik, A. N., Mikhael, J. G., Bech, P., Tsutsui-Kimura, I., Sun, F., Zhang, Y., Li, Y., Watabe-Uchida, M., Gershman, S. J., et al. (2020). A unified framework for dopamine signals across timescales. Cell, 183(6):1600-1616.

Kishida, K. T., Saez, I., Lohrenz, T., Witcher, M. R., Laxton, A. W., Tatter, S. B., White, J. P., Ellis, T. L., Phillips, P. E., and Montague, P. R. (2016). Subsecond dopamine fluctuations in human striatum encode superposed error signals about actual and counterfactual reward. Proceedings of the National Academy of Sciences, 113(1):200-205.

Krueger, K. A. and Dayan, P. (2009). Flexible shaping: How learning in small steps helps. Cognition, 110(3):380-394.

Ludvig, E. A., Sutton, R. S., and Kehoe, E. J. (2012). Evaluating the td model of classical conditioning. Learning $\mathcal{E}$ behavior, 40(3):305-319. 
Mackintosh, N. J. (1983). Conditioning and associative learning. Clarendon Press Oxford.

McClure, S. M., Daw, N. D., and Montague, P. R. (2003). A computational substrate for incentive salience. Trends in neurosciences, 26(8):423-428.

McCutcheon, J. E. (2015). The role of dopamine in the pursuit of nutritional value. Physiology $\mathcal{E}$ behavior, 152:408-415.

McCutcheon, J. E., Beeler, J. A., and Roitman, M. F. (2012). Sucrose-predictive cues evoke greater phasic dopamine release than saccharin-predictive cues. Synapse, 66(4):346-351.

Mehiel, R. and Bolles, R. C. (1984). Learned flavor preferences based on caloric outcome. Animal Learning \& Behavior, 12(4):421-427.

Mehiel, R. and Bolles, R. C. (1988). Hedonic shift learning based on calories. Bulletin of the Psychonomic Society, 26(5):459-462.

Montague, P. R., Dayan, P., and Sejnowski, T. J. (1996). A framework for mesencephalic dopamine systems based on predictive hebbian learning. Journal of neuroscience, 16(5):1936-1947.

Morales, I. and Berridge, K. C. (2020). 'liking'and 'wanting'in eating and food reward: Brain mechanisms and clinical implications. Physiology E behavior, page 113152.

Myers, K. P. (2018). The convergence of psychology and neurobiology in flavor-nutrient learning. Appetite, 122:36-43.

Myers, K. P. and Sclafani, A. (2001). Conditioned enhancement of flavor evaluation reinforced by intragastric glucose: Ii. taste reactivity analysis. Physiology E behavior, 74(45):495-505.

Myers, K. P. and Sclafani, A. (2003). Conditioned acceptance and preference but not altered taste reactivity responses to bitter and sour flavors paired with intragastric glucose infusion. Physiology $\mathcal{E}$ behavior, 78(2):173-183.

Myers, K. P., Summers, M. Y., Geyer-Roberts, E., and Schier, L. A. (2020). The role of post-ingestive feedback in the development of an enhanced appetite for the orosensory properties of glucose over fructose in rats. Nutrients, 12(3):807.

$\mathrm{Ng}$, A. Y., Harada, D., and Russell, S. (1999). Policy invariance under reward transformations: Theory and application to reward shaping. In Icml, volume 99, pages 278-287.

Niv, Y., Daw, N. D., Joel, D., and Dayan, P. (2007). Tonic dopamine: opportunity costs and the control of response vigor. Psychopharmacology, 191(3):507-520.

Oudeyer, P.-Y., Kaplan, F., and Hafner, V. V. (2007). Intrinsic motivation systems for autonomous mental development. IEEE transactions on evolutionary computation, 11(2):265-286.

Petzschner, F. H., Garfinkel, S. N., Paulus, M. P., Koch, C., and Khalsa, S. S. (2021). Computational models of interoception and body regulation. Trends in neurosciences, 44(1):63-76.

Randløv, J. and Alstrøm, P. (1998). Learning to drive a bicycle using reinforcement learning and shaping. In ICML, volume 98, pages 463-471. Citeseer. 
Reber, R., Schwarz, N., and Winkielman, P. (2004). Processing fluency and aesthetic pleasure: is beauty in the perceiver's processing experience? Personality and Social Psychology Review, 8(4):364-382.

Ren, X., Ferreira, J. G., Zhou, L., Shammah-Lagnado, S. J., Yeckel, C. W., and de Araujo, I. E. (2010). Nutrient selection in the absence of taste receptor signaling. Journal of Neuroscience, 30(23):8012-8023.

Ryali, C. K., Goffin, S., Winkielman, P., and Yu, A. J. (2020). From likely to likable: The role of statistical typicality in human social assessment of faces. Proceedings of the National Academy of Sciences of the United States of America, 117(47):29371-29380.

Salamone, J. D. and Correa, M. (2013). Dopamine and food addiction: lexicon badly needed. Biological psychiatry, 73(9):e15-e24.

Schmidhuber, J. (2010). Formal theory of creativity, fun, and intrinsic motivation (1990-2010). IEEE transactions on autonomous mental development, 2(3):230-247.

Schultz, W., Dayan, P., and Montague, P. R. (1997). A neural substrate of prediction and reward. Science, 275(5306):1593-1599.

Sclafani, A. (2001). Post-ingestive positive controls of ingestive behavior. Appetite, 36(1):79-83.

Sclafani, A. (2004). Oral and postoral determinants of food reward. Physiology \& behavior, 81(5):773-779.

Singh, S., Lewis, R. L., and Barto, A. G. (2009). Where do rewards come from. In Proceedings of the annual conference of the cognitive science society, pages 2601-2606. Cognitive Science Society.

Skinner, B. (1938). The Behavior of Organisms. BF Skinner Foundation.

Skinner, B. F. (1975). The shaping of phylogenic behavior. Journal of the Experimental Analysis of Behavior, 24(1):117-120.

Sorg, J., Singh, S. P., and Lewis, R. L. (2010). Internal rewards mitigate agent boundedness. In ICML.

Suri, R. E. and Schultz, W. (1999). A neural network model with dopamine-like reinforcement signal that learns a spatial delayed response task. Neuroscience, 91(3):871-890.

Sutton, R. S. (1988). Learning to predict by the methods of temporal differences. Machine learning, 3(1):9-44.

Sutton, R. S. and Barto, A. G. (1998). Reinforcement Learning: An Introduction. MIT Press, Cambridge, MA.

Tellez, L. A., Han, W., Zhang, X., Ferreira, T. L., Perez, I. O., Shammah-Lagnado, S. J., Van Den Pol, A. N., and de Araujo, I. E. (2016). Separate circuitries encode the hedonic and nutritional values of sugar. Nature neuroscience, 19(3):465-470.

Van de Cruys, S. and Wagemans, J. (2011). Putting reward in art: A tentative prediction error account of visual art. i-Perception, 2(9):1035-1062.

Veldhuizen, M. G., Babbs, R. K., Patel, B., Fobbs, W., Kroemer, N. B., Garcia, E., Yeomans, M. R., and Small, D. M. (2017). Integration of sweet taste and metabolism determines carbohydrate reward. Current Biology, 27(16):2476-2485. 
Warlow, S. M., Naffziger, E. E., and Berridge, K. C. (2020). The central amygdala recruits mesocorticolimbic circuitry for pursuit of reward or pain. Nature communications, 11(1):1-15.

Wiewiora, E. (2003). Potential-based shaping and q-value initialization are equivalent. Journal of Artificial Intelligence Research, 19:205-208. 